\title{
Avaliação da estratégia saúde da família a partir das crenças dos profissionais ${ }^{1}$
}

\author{
Evaluation of family health strategy based \\ on the beliefs of professionals
}

\author{
Francisco José Batista de ALBUQUERQUE ${ }^{2}$ \\ Cynthia de Freitas MELO $^{3}$ \\ Francisco Elmiro de SOUZA FILHO ${ }^{4}$ \\ João Lins de ARAÚJO NETO5
}

\section{Resumo}

As pesquisas de avaliação da Estratégia Saúde da Família funcionam como ferramenta de feedback nas decisões de seus gestores. Assim, o presente estudo objetivou avaliá-la a partir das crenças dos seus profissionais. Uma amostra probabilística de 337 profissionais respondeu à "Escala de Avaliação da Estratégia Saúde da Família pelos Profissionais", que investiga os seguintes fatores: 1) Recursos materiais $(\alpha=0,86)$; 2) Eficiência no atendimento $(\alpha=0,80)$; e 3$)$ Infraestrutura física $(\alpha=0,73)$. Os resultados apresentaram uma avaliação positiva dos recursos materiais por $76,7 \%$ dos profissionais. A eficiência do atendimento foi avaliada negativamente por $53,4 \%$ dos participantes e a infraestrutura foi avaliada positivamente por $71,2 \%$ dos profissionais. Constatou-se ainda que todos os fatores foram pior avaliados pelos profissionais de nível médio/técnico. Conclui-se que existem aspectos positivos na estratégia, e que eles devem ser usados como modelos replicáveis, ressaltando-se, entretanto, entraves que minimizam sua eficácia e que precisam ser corrigidos objetivando melhorar as condições de prestação de serviço para seus usuários.

Unitermos: Crenças. Estratégia saúde da família. Pesquisas de avaliação.

\begin{abstract}
The evaluation research on Family Health Strategy works as a feedback tool for the decisions of its managers. Therefore, the present study aimed to evaluate it from the perspective of the beliefs of its professionals. A random sample of 337 professionals answered the "Family Health Strategy Rating Scale by professionals", which investigates the following factors: 1) Material resources $(\alpha=0.86), 2)$ Efficiency of care ( $\alpha=0.80)$, and 3) Physical infrastructure $(\alpha=0.73)$. The results showed a positive assessment of the material resources by $76.7 \%$ of the professionals. The efficiency of care was rated negatively by $53.4 \%$ of participants and the infrastructure was evaluated positively by $71.2 \%$ of the professionals. It was further observed that all factors resulted in inferior ratings from the medium/technical level professionals. It was concluded that there are positive aspects in the strategy, and these should be used as models for replication, but emphasizing, however, there are impediments that minimize its effectiveness, and that need to be fixed with the aim of improving the conditions of service providing to its users.
\end{abstract}

Uniterms: Beliefs. Family health strategy. Evaluation researches.

$\boldsymbol{\nabla} \mathbf{v} \boldsymbol{\nabla} \mathbf{v}$

1 Artigo elaborado a partir da dissertação de C.F. MELO, intitulada "Avaliação da estratégia saúde da família a partir das crenças de seus profissionais". Universidade Federal da Paraíba, 2009.

2 Universidade Estadual da Paraíba, Centro de Ciências Médicas. Campus I, Bairro Universitário, 58429-500, Campina Grande, PB, Brasil. Correspondência para/Correspondence to: F.J.B. ALBUQUERQUE.E-mail: <frajoba@uol.com.br>.

3 Universidade Federal do Rio Grande do Norte, Centro de Ciências Humanas. Natal, RN, Brasil.

${ }^{4}$ Universidade Federal da Paraíba, Centro de Ciências Exatas e da Natureza. João Pessoa, PB, Brasil.

${ }^{5}$ Universidade Federal da Paraíba, Centro de Ciências Médicas. João Pessoa, PB, Brasil. 
Este estudo objetivou avaliar a Estratégia Saúde da Família (ESF) em João Pessoa, Paraíba, a partir das crenças dos seus profissionais, buscando compreender os fatores que influenciam o desempenho efetivo do trabalho dos profissionais da Equipe de Saúde da Família (EqSF), e possibilitando o êxito ou o fracasso da estratégia. Acredita-se que João Pessoa, a exemplo de outras cidades de porte médio do Brasil, deva ser mais contemplada pelas pesquisas científicas, pois fica à margem da produção do conhecimento nacional, uma vez que a maioria dos estudos é realizada nas grandes cidades.

O Programa Saúde da Família (PSF), como era chamado até 2006, assim como os outros programas sociais brasileiros, surgiu a partir da demanda da população. Como projetos governamentais, deveriam ser tratados como experimentos planejados que objetivam atingir uma mudança na realidade social (Campbell, 1969; Cano, 2004). Sabe-se, todavia, que esses programas são criados e implantados por tentativa e erro. Contudo, a população, que paga os serviços, acompanha uma prática cotidiana que se diferencia e se distancia das formulações teóricas e legislativas, resultando em descontinuidade e repetições de erros que poderiam ser substituídos pelas boas práticas que pudessem ser encontradas pelos processos de avaliação dos programas.

Cada vez mais, entretanto, grupos de pesquisadores, concorrentes políticos e mídia exigem prestações de contas, não apenas financeiras, mas também dos resultados dessas ações. A população, cada vez mais consciente de sua posição de pagante de taxas e impostos e de "sujeitos de direitos", reclama, no cotidiano de funcionamento, a resolução das ações governamentais: marcação cerrada, que "exige" a avaliação contínua da eficácia, eficiência e efetividade das políticas públicas.

Nesse cenário, a avaliação de programas funciona como uma ferramenta fundamental para auxiliar, através de feedback, nas decisões dos gestores no tocante à implementação, ao processo e aos resultados alcançados, informações sistemáticas que podem ser utilizadas nos seus aprimoramentos.

De acordo com Belloni, Magalhães e Sousa (2003), existem, entretanto, poucas experiências de avaliação sistemática de desempenho e resultados dos programas. A maioria dos estudos prioriza informações sobre o impacto, esquecendo-se das consequências mais amplas e difusas das ações.
Guiado por esse propósito, o presente estudo realizou uma avaliação da ESF sob a perspectiva de seus profissionais, que são os operadores da estratégia. Eles se tornam, assim, fonte preciosa de informação, pois, além de conhecerem a realidade do cotidiano de funcionamento da estratégia, são sujeitos que também possuem o poder de modificá-la. Sabe-se, pois, que a operacionalização modifica as políticas públicas, tal como concebida por seus formuladores (Barreira, 2001).

Em geral, os usuários da ESF são os maiores focos nesse tipo de pesquisa, pois são eles que sofrem as reformas e a ação que se exerce sobre eles, as doenças e as mudanças organizacionais, a espera e a desinformação, atuando verdadeiramente como os "sujeitos-pacientes". São eles o foco dos trabalhos de Oliveira (2006), que já escutaram as queixas dos usuários da Estratégia Saúde da Família em João Pessoa, detectando suas falhas operacionais.

Nesse contexto de dificuldades, entretanto, não há como negar a existência de "funcionários-missionários", que, além de lidarem com os pacientes que muitas vezes os consideram maximizadores do seu stress (Sousa \& Souza Filho, 2008), e com as dificuldades do sistema, tentam superar os problemas para atender a população (Faleiros, 2003). Cresce a demanda de atendimentos, e o serviço torna-se impermeável para muitas pessoas que dele necessitam e que a ele têm direito. Para essas pessoas, não interessa de qual esfera do governo provém o serviço, e sim se elas são eficientemente atendidas na hora da necessidade. Se não ocorre tudo como esperado, os profissionais que estão nas Unidades de Saúde da Família (USF) tornam-se os únicos alvos visíveis para as queixas e críticas dos usuários, sendo aqueles responsabilizados por todas as deficiências da estratégia. Por esse motivo, contempla-se a importância de pesquisas de avaliação da ESF que foquem também os profissionais que atuam no cotidiano de funcionamento da estratégia.

De acordo com Albuquerque (1999), entretanto, os psicólogos brasileiros têm pouco interesse em relacionar o conhecimento em avaliação de comportamento com avaliação de políticas públicas, embora sejam conhecedores de conteúdos sobre relações intergrupais, crenças coletivas, atitudes, comportamentos individuais e grupais, psicometria, entre outras ferramentas fundamentais para esse tipo de tarefa. 
Tendo esse referencial teórico como pano de fundo, buscou-se conhecer as crenças dos profissionais sobre fatores específicos da ESF: recursos materiais (insumos, vacinas e medicação), eficiência no atendimento (sistema de referência/contra-referência e capacitação profissional) e infraestrutura física. Dessa forma, apreenderam-se os aspectos positivos, falhas e limitações do seu funcionamento.

Abordaram-se os profissionais da ESF sob o aspecto cognitivo, utilizando a teoria das crenças, pois, segundo a teoria cognitivo-comportamental (Beck, 1982; Cordioli, 2008), a partir das experiências dentro das USF, esses profissionais formam crenças sobre a estratégia, e essas afetam seu estado afetivo e seu padrão comportamental. Desse modo, a apreensão dessas crenças possibilitou a compreensão das condições de prestações de serviços para a comunidade, sob a óptica de seus profissionais, permitindo-nos, assim, detectar os aspectos cruciais que conduzem sua atuação na ESF.

\section{Método}

A presente pesquisa objetivou avaliar a Estratégia Saúde da Família (ESF) em João Pessoa, Paraíba, a partir das crenças dos profissionais da Equipe de Saúde da Família (EqSF), com o intuito de compreender as condições e os fatores que influenciam o desempenho efetivo do trabalho dessas equipes na ESF, possibilitando seu êxito ou fracasso. Assim, por meio desses sujeitos que implementam a ESF, pretende-se conhecer o "SUS real", tal como ele acontece no cotidiano de atenção à saúde.
O presente estudo se configura como uma pesquisa descritiva correlacional aplicada, cujas variáveis antecedentes referem-se às condições de trabalho nas Unidades de Saúde da Família (USF) e ao perfil dos participantes da pesquisa, enquanto as variáveis consequentes referem-se à avaliação que os profissionais das equipes multidisciplinares mínimas da ESF fazem da estratégia.

\section{Participantes}

Foi utilizada uma amostragem probabilística estratificada, por categoria profissional e por distrito sanitário, composta por 337 profissionais das EqSF (médico, dentistas, enfermeiros, auxiliares de enfermagem, Auxiliares de Consultório Dentário - ACD e Agentes Comunitários de Saúde - ACS), de forma que eles fossem efetivamente representantes dos 2095 profissionais que atuam no município (Tabela 1).

\section{Instrumentos}

O uso da Escala de Avaliação da ESF pelos profissionais (Melo 2009) objetivou verificar as crenças dos profissionais sobre diversos aspectos da ESF. A escala tem 24 itens e quatro pontos de resposta (nunca, raramente, frequentemente e sempre), e investiga os seguintes fatores: 1$)$ Recursos materiais $(\alpha=0,86)$, com 10 itens; 2) Eficiência no atendimento $(\alpha=0,80)$, com 8 itens; e 3) Infraestrutura física $(\alpha=0,73)$, com 6 itens.

\section{Procedimentos}

O instrumento, utilizado forma individual, é autoaplicável dentro das USF. Quanto aos aspectos

Tabela 1.Número de sujeitos da população e participantes da amostra, segundo agrupamento estratificado de categoria profissional e distrito sanitário. João Pessoa (PB), 2009.

\begin{tabular}{|c|c|c|c|c|c|c|}
\hline \multirow{3}{*}{ Categoria profissional } & \multicolumn{5}{|c|}{ População (amostra) (n) } & \multirow{3}{*}{ Total } \\
\hline & \multicolumn{5}{|c|}{ Distrito } & \\
\hline & Sanitário I & Sanitário II & Sanitário III & Sanitário IV & Sanitário V & \\
\hline Médico & $46(8)$ & $37(6)$ & $54(9)$ & $26(4)$ & $16(3)$ & 30 \\
\hline Dentistas & $47(8)$ & $37(6)$ & $54(9)$ & $27(5)$ & $18(3)$ & 31 \\
\hline ACD & $46(8)$ & $37(6)$ & $54(9)$ & $27(5)$ & $18(3)$ & 31 \\
\hline Enfermeiros & $47(8)$ & $37(6)$ & $53(9)$ & $26(4)$ & $19(3)$ & 30 \\
\hline Auxiliares de enfermagem & $46(8)$ & $39(6)$ & $53(9)$ & $26(4)$ & $18(3)$ & 30 \\
\hline ACS & $314(89)$ & $245(6)$ & $370(57)$ & $152(24)$ & $106(17)$ & 185 \\
\hline Total & $546(89)$ & $432(68)$ & $638(102)$ & $284(46)$ & $195(32)$ & 337 \\
\hline
\end{tabular}

ACD: auxiliar de consultório dentário; ACS: agentes comunitários de saúde. 
éticos referentes a pesquisas envolvendo seres humanos, o estudo foi aprovado por comitê de ética da Universidade Federal da Paraíba, sob o protocolo de n0298, em 09/07/2008. Os participantes foram informados previamente objetivos e procedimentos do estudo, e foram-Ihes garantidos o anonimato da sua colaboração e a confidencialidade de suas respostas. Foi solicitado, ainda, que eles assinassem um Termo de Consentimento Livre e Esclarecido.

Como os dados foram coletados por um instrumento com características ordinais, optou-se por considerar a estatística não paramétrica como a mais idônea para a exploração dos dados, pois a utilização da média pode gerar distorções na interpretação dos resultados.

A análise de dados aconteceu em três etapas. Na primeira, foram realizadas as frequências e as porcentagens dos índices gerais dos fatores. Em seguida, foram obtidas, ainda, as frequências e porcentagens de cada item dos fatores. Por fim, na terceira etapa, foram realizadas comparações entre os profissionais por escolaridade (nível médio e técnico x superior) e por categoria profissional, por meio do qui-quadrado.

\section{Resultados e Discussão}

Serão apresentados nesta seção os resultados encontrados em cada um dos fatores anteriormente descritos: 1) Recursos materiais; 2) Eficiência no atendimento; e 3) Infraestrutura física.
Os resultados serão apresentados pela porcentagem cumulativa das frequências das avaliações negativas ("nunca" e "raramente") e positivas ("frequentemente" e "sempre") de cada item dos fatores, o que permite a obtenção de dados mais detalhados sobre a distribuição das crenças dos respondentes sobre os diversos fatores. Além disso, serão apresentadas as comparações das avaliações de cada fator por nível de escolaridade dos profissionais e por categoria profissional.

\section{Recursos materiais (Fator 1)}

Os itens que compõem esse fator refletem as crenças que os profissionais da ESF têm sobre a disponibilidade de insumos para realização de seu trabalho (luvas, gazes, máscaras, amálgama, seringas); de vacinas para a imunização; e de remédios e contraceptivos para distribuir aos usuários.

Nas análises gerais desse fator, observou-se que 76,7\% dos participantes da pesquisa Ihe atribuíram uma avaliação global positiva. Com um olhar mais cuidadoso sobre os resultados obtidos, analisaram-se as frequências de respostas de cada item do fator. Pôde-se, dessa forma, identificar a avaliação da disponibilidade de cada recurso material analisado e verificar os dados que se camuflam por trás do índice de 76,7\% de avaliações positivas. Verificou-se que, embora pareçam números pequenos, os percentuais cumulativos dos pontos "nunca disponível"e"raramente disponível"- avaliação negativa - apresentam dados preocupantes, se for levado em consideração que 23,3\% dos profissionais de diferentes USF da

Tabela 2. Distribuição da porcentagem de respostas dos profissionais da EqSF sobre a disponibilidade dos recursos materiais que compõem o fator 1. João Pessoa (PB), 2009.

\begin{tabular}{|c|c|c|c|c|c|c|c|}
\hline & \multirow[b]{2}{*}{ Recursos materiais } & \multicolumn{6}{|c|}{ Percentual (\%) } \\
\hline & & $\begin{array}{c}\text { Nunca } \\
\text { disponível }\end{array}$ & $\begin{array}{l}\text { Raramente } \\
\text { disponível }\end{array}$ & $\begin{array}{c}\% \\
\text { acumulado }\end{array}$ & $\begin{array}{c}\text { Frequentemente } \\
\text { disponível }\end{array}$ & $\begin{array}{c}\text { Sempre } \\
\text { disponível }\end{array}$ & $\begin{array}{c}\% \\
\text { acumulado }\end{array}$ \\
\hline & Luvas & 3,60 & 13,10 & 16,70 & 50,70 & 32,30 & 83,30 \\
\hline \multirow{8}{*}{ 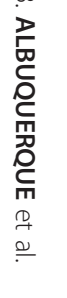 } & Gazes & 9,20 & 27,60 & 36,80 & 47,80 & 15,40 & 63,20 \\
\hline & Máscaras & 4,20 & 10,70 & 15,00 & 50,40 & 33,80 & 85,00 \\
\hline & Amálgama para o dentista & 2,50 & 9,10 & 11,60 & 45,60 & 42,80 & 88,40 \\
\hline & Seringas descartáveis & 3,30 & 13,40 & 16,70 & 45,60 & 37,70 & 83,30 \\
\hline & Vacinas & 3,00 & 11,70 & 14,70 & 69,10 & 16,20 & 85,30 \\
\hline & Anestesia & 4,50 & 7,70 & 12,20 & 52,30 & 35,50 & 87,80 \\
\hline & Remédios & 8,70 & 51,10 & 59,80 & 39,00 & 1,20 & 40,20 \\
\hline & Camisinhas & 4,50 & 21,60 & 26,00 & 56,30 & 17,70 & 74,00 \\
\hline 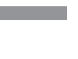 & Anticoncepcionais & 5,10 & 18,60 & 23,70 & 65,80 & 10,50 & 76,30 \\
\hline 365 & Média porcentual & 4,86 & 18,46 & 23,32 & 52,26 & 24,31 & 76,68 \\
\hline
\end{tabular}


cidade afirmam que os recursos materiais "nunca" ou "raramente" estão disponíveis. Mais especificamente, 36,8\% dos entrevistados afirmaram que não possuem gazes, e 59,8\% dos profissionais afirmaram que não possuem remédios disponíveis para a comunidade (Tabela 2).

Ao se compararem as pontuações desse fator por grupo de escolaridade dos profissionais e ao se analisarem as porcentagens cumulativas das frequências das avaliações positivas e negativas, observou-se que os profissionais de nível médio e técnico apresentam maior frequência de avaliação negativa $(f=60,59 ; 24,63 \%)$ em relação aos de nível superior ( $f=17,95 ; 19,73 \%)$, com diferença estatisticamente significativa $\left(\chi^{2}=71,98 ; 1 \mathrm{gl}\right.$, $p \leq 0,05)$.

Por fim, foram realizadas comparações das avaliações do primeiro fator entre as seis categorias profissionais, e observou-se que há diferença estatisticamente significativa entre elas $\left(\chi^{2}=975,24 ; 5 \mathrm{gl}, p \leq 0,05\right)$.

Enfatiza-se que os agentes comunitários de saúde foram os profissionais que apresentaram mais avaliações negativas ( $f=480 ; 25,95 \%)$, provavelmente por serem a eles que mais são solicitados os recursos (contraceptivos e remédios), uma vez que estão em contato mais direto com as demandas populacionais, como sugere Oliveira (2006).

\section{Eficiência no atendimento (Fator 2)}

Esse fator refere-se aos aspectos organizacionais das Unidades de Saúde da Família, que abrangem o acesso da comunidade à ESF, que verifica, sob a perspectiva dos profissionais, se o atendimento na ESF funciona ou não. Divide-se em dois subfatores: "sistema de referência e contra-referência", que abrange os encaminhamentos para outros níveis de complexidade; e "capacitação profissional", que reflete as crenças dos profissionais da ESF sobre as capacitações e treinamentos oferecidos aos profissionais da ESF, antes de começarem a trabalhar na ESF e durante o exercício de sua profissão.

Obteve-se como resultado da avaliação do fator geral "eficiência do atendimento" uma avaliação negativa por $53,4 \%$ dos profissionais das ESF.

Ao se analisarem mais detalhadamente seus subfatores, averiguou-se que o "sistema de referência e contra-referência" foi avaliado negativamente por 63,5\% dos entrevistados. Tais dados são preocupantes quando se verifica que $51,6 \%$ dos profissionais afirmaram que a central de marcação não é eficiente; 68,5\% afirmaram não haver vagas suficientes para os outros níveis; $73,0 \%$ acreditam que há falhas na contra-referência e 61,1\% afirmam que a referência para níveis de saúde mais complexos não é satisfatória, dados que confirmam o estudo de Melo (2009) (Tabela 3).

A análise mais detalhada do subfator "capacitação profissional" constatou que uma quantidade considerável de entrevistados (42,9\%) Ihe atribuía avaliações negativas ("nunca" ou "raramente"). Dessa forma, torna-se inquietante verificar que 32,6\% dos profissionais afirmaram que "nunca" ou "raramente" são capacitados; 26,1\% afirmaram não haver reuniões da equipe para discutir os casos dos pacientes; 51,9\% dos profissionais afirmam que os profissionais contratados não são treinados para o trabalho com a comunidade; e que 61,1\% afirmam que a Secretaria de Saúde não oferece cursos de capacitação para os profissionais (Tabela 3).

Compararam-se ainda as frequências cumulativas das avaliações positivas e negativas por grupos de escolaridade, observando-se que os profissionais de nível médio e técnico apresentam menor frequência de avaliação negativa ( $f=131,07 ; 53,28 \%$ ) do que os profissionais de nível superior ( $f=58,89 ; 54,82 \%$ ), com uma diferença estatisticamente significativa $\left(\chi^{2}=45,20 ; 1 \mathrm{gl}\right.$, $p \leq 0,05)$. Esse fato pode ser explicado pela exigência do Ministério da Saúde de que todos os ACS's façam cursos preparatórios antes de serem inseridos na ESF, sendo essa categoria, portanto, mais privilegiada pelas políticas de capacitações.

Por fim, foram comparadas as avaliações do fator entre as seis categorias profissionais, detectando-se diferenças estatisticamente significativas entre elas $\left(\chi^{2}=34,74 ; 5 \mathrm{gl}, p \leq 0,05\right)$. Verificou-se que a pior avaliação também foi feita pelos agentes comunitários de saúde $(f=800 ; 54,05 \%)$, provavelmente porque são esses profissionais que mais escutam as queixas dos usuários sobre a eficiência no atendimento.

\section{Infraestrutura física e material (Fator 3)}

Esse fator é composto por itens que refletem o estado das USF no que se refere aos aspectos de 
Tabela 3. Distribuição da porcentagem de respostas dos profissionais da EqSF sobre o sistema de referência/contra-referência e capacitação profissional. João Pessoa (PB), 2009.

\begin{tabular}{|c|c|c|c|c|c|c|}
\hline \multirow{2}{*}{$\begin{array}{l}\text { Itens do Subfator Sistema de referência e } \\
\text { contra-referência }\end{array}$} & \multicolumn{6}{|c|}{ Percentual (\%) } \\
\hline & Nunca & Raramente & $\%$ acumulado & Frequentemente & Sempre & $\%$ acumulado \\
\hline $\begin{array}{l}\text { A central de marcação para o encaminhamento dos } \\
\text { pacientes é eficiente }\end{array}$ & 15,70 & 35,90 & 51,60 & 36,80 & 11,00 & 48,40 \\
\hline $\begin{array}{l}\text { Existem vagas suficientes para o encaminhamento dos } \\
\text { pacientes para médicos especialistas e exames }\end{array}$ & 13,60 & 54,90 & 68,50 & 27,60 & 3,60 & 31,50 \\
\hline $\begin{array}{l}\text { A contra-referência (retorno ao PSF dos pacientes que } \\
\text { foram encaminhados para atendimentos em níveis de } \\
\text { saúde mais complexos) é satisfatória }\end{array}$ & 27,60 & 45,40 & 73,00 & 17,50 & 9,50 & 27,00 \\
\hline $\begin{array}{l}\text { A referência para atendimentos em níveis de saúde mais } \\
\text { complexos é satisfatória }\end{array}$ & 13,90 & 47,20 & 61,10 & 33,50 & 5,30 & 38,90 \\
\hline Média porcentual & 17,70 & 45,85 & 63,55 & 28,85 & 29,40 & 36,45 \\
\hline \multirow{2}{*}{$\begin{array}{l}\text { Itens do Subfator Sistema de referência e } \\
\text { contra-referência }\end{array}$} & \multicolumn{6}{|c|}{ Percentual (\%) } \\
\hline & Nunca & Raramente & $\%$ acumulado & Frequentemente & Sempre & $\%$ acumulado \\
\hline $\begin{array}{l}\text { Fui capacitado(a) pela Secretaria de Saúde para o tra- } \\
\text { balho que realizo no PSF }\end{array}$ & 6,80 & 25,80 & 32,60 & 34,70 & 32,70 & 67,40 \\
\hline $\begin{array}{l}\text { A equipe do PSF se reúne para discutir os casos dos } \\
\text { pacientes }\end{array}$ & 5,60 & 20,50 & 26,10 & 33,80 & 40,10 & 73,90 \\
\hline $\begin{array}{l}\text { Os profissionais contratados para atuar no PSF são } \\
\text { treinados para trabalhar com a comunidade }\end{array}$ & 12,80 & 39,20 & 51,90 & 28,80 & 19,30 & 48,10 \\
\hline $\begin{array}{l}\text { A Secretaria de Saúde oferece cursos de capacitação } \\
\text { para os profissionais do PSF }\end{array}$ & 8,30 & 52,80 & 61,10 & 25,80 & 13,10 & 38,90 \\
\hline Média Porcentual & 8,37 & 34,58 & 42,92 & 30,78 & 26,30 & 57,08 \\
\hline
\end{tabular}

PSF: programa saúde da família; EqSF: equipe de saúde da família.

infraestrutura física e das condições de materiais permanentes (instrumentos e equipamentos) disponibilizados pelos gestores para a otimização do trabalho dos profissionais da ESF no oferecimento dos serviços de saúde aos seus usuários.

$\mathrm{Na}$ análise geral do fator, verificou-se uma aprovação da infraestrutura por $71,2 \%$ dos profissionais da equipe de saúde da família. Enfatiza-se que, embora a cidade tenha criado novas unidades unificadas, com excelente estrutura, apresentando avaliações positivas, ainda existem diversas unidades precárias, sem a mínima infraestrutura. Os dados da presente pesquisa mostram, portanto, que ainda há $28,8 \%$ de reprovações. Assim, 47,2\% dos entrevistados afirmaram que "nunca" ou "raramente" os instrumentos existem em quantidade suficiente; $37,4 \%$ dos profissionais afirmam que as condições do prédio não são boas; e 25,2\% responderam que os atendimentos não são feitos em salas adequadas

368 (Tabela 4).
Ao comparar a avaliação do fator infraestrutura física entre os grupos de diferentes níveis de escolaridade, verificou-se que os profissionais de nível médio e técnico apresentam maior frequência de avaliação negativa $(f=77,37 ; 31,45 \%)$ do que os de nível superior $(f=24,52 ; 21,63 \%)$, com diferença estatisticamente significativa $\left(\chi^{2}=58,14 ; 1 \mathrm{gl}, p \leq 0,05\right)$.

Quanto à avaliação da infraestrutura física entre os seis grupos profissionais, observa-se que há diferença estatisticamente significativa $\left(\chi^{2}=366,28 ; 5 \mathrm{gl}, p \leq 0,05\right)$, tendo sido os agentes comunitários de saúde ( $f=370$; $33,33 \%$ ) e os auxiliares de enfermagem ( $f=58 ; 32,22 \%$ ) os profissionais que mais negativamente a avaliaram.

Por fim, contempla-se que, apesar de a maioria $(60,5 \%)$ dos profissionais ser concursada, existe um alto índice de médicos e enfermeiros que são prestadores de serviço: $80 \%$ em ambas as categorias $\left[\chi^{2}(2)=181,75\right.$; $p<0,05]$, sendo os auxiliares de enfermagem $(63,3 \%)$ e ACS's $(71,9 \%)$ as categorias com mais profissionais concursados (Tabela 5). 
Tabela 4. Distribuição da porcentagem de respostas dos profissionais da EqSF sobre os itens que compõem o fator 3. João Pessoa (PB), 2009.

\begin{tabular}{|c|c|c|c|c|c|c|}
\hline \multirow[b]{2}{*}{ Itens do Fator 3: Infraestrutura física } & \multicolumn{6}{|c|}{ Percentual (\%) } \\
\hline & $\begin{array}{c}\text { Nunca } \\
\text { disponível }\end{array}$ & $\begin{array}{l}\text { Raramente } \\
\text { disponível }\end{array}$ & $\begin{array}{c}\% \\
\text { acumulado }\end{array}$ & $\begin{array}{l}\text { Frequentemente } \\
\text { disponível }\end{array}$ & $\begin{array}{l}\text { Sempre } \\
\text { disponível }\end{array}$ & $\begin{array}{c}\% \\
\text { acumulado }\end{array}$ \\
\hline $\begin{array}{l}\text { Neste seu local de trabalho, os atendimentos são feitos } \\
\text { em salas adequadas }\end{array}$ & 11,60 & 13,60 & 25,20 & 27,90 & 46,90 & 74,80 \\
\hline $\begin{array}{l}\text { Os equipamentos (cadeira do dentista, balança) da USF } \\
\text { (PSF) estão em boas condições de uso }\end{array}$ & 0,90 & 13,40 & 14,20 & 40,40 & 45,40 & 85,80 \\
\hline $\begin{array}{l}\text { Os instrumentos (estetoscópio, prancheta, termômetro) } \\
\text { da USF (PSF) existem em quantidade suficiente }\end{array}$ & 16,90 & 30,30 & 47,20 & 30,60 & 22,30 & 52,80 \\
\hline $\begin{array}{l}\text { As condições do prédio da USF (PSF) em que trabalho } \\
\text { são adequadas }\end{array}$ & 18,40 & 19,00 & 37,40 & 21,10 & 41,50 & 62,60 \\
\hline $\begin{array}{l}\text { Os equipamentos (cadeira do dentista, balança) da USF } \\
\text { (PSF) existem em quantidade suficiente }\end{array}$ & 6,80 & 14,80 & 21,70 & 32,9 & 45,40 & 78,30 \\
\hline $\begin{array}{l}\text { Os instrumentos (estetoscópio, prancheta, termômetro) } \\
\text { que eu utilizo na USF (PSF) estão em boas condições de } \\
\text { uso }\end{array}$ & 2,70 & 24,60 & 27,30 & 43,90 & 28,80 & 72,70 \\
\hline Total & 9,55 & 19,28 & 28,83 & 32,8 & 38,37 & 71,17 \\
\hline
\end{tabular}

EqSF: equipe de saúde da família; USF: unidades de saúde da família; PSF: programa saúde da família.

Tabela 5. Tipo de vínculo empregatício dos profissionais da Equipe de Saúde da Família segundo categoria profissional (n=337). João Pessoa (PB), 2009

\begin{tabular}{lccccccc}
\hline Variáveis & Dados estatísticos & Médico & Enfermeira & Auxiliar de enfermagem & Dentista & ACD & ACS \\
\hline Concursado & $\mathrm{F}$ & 6 & 6 & 19 & 13 & 11 & 13 \\
& $\%$ & 20,0 & 20,0 & 63,3 & 41,9 & 35,5 & 71,9 \\
Serviço prestado & $\mathrm{F}$ & 24 & 24 & 11 & 18 & 20 & 52 \\
& $\%$ & 80,0 & 80,0 & 36,7 & 58,1 & 64,5 & 28,1 \\
\hline
\end{tabular}

ACD: auxiliar de consultório dentário; ACS: agentes comunitários de saúde.

\section{Consideração Finais}

O Brasil não tem a cultura desenvolvida de avaliação sobre a qualidade dos serviços públicos oferecidos, especialmente os voltados para programas sociais na área de saúde, que são idealizados e implementados, sem que na maioria das vezes seus resultados sejam avaliados. Impera a distância entre os princípios que os regulamentam e aqueles que os gerenciam, e destes com o funcionamento cotidiano do programa junto à sociedade. Como detentores de ferramentas capazes de auxiliar esses procedimentos avaliativos, os psicólogos brasileiros devem se interessar em relacionar o conhecimento em avaliação de comportamento com avaliação de políticas públicas.

A Estratégia Saúde da Família é uma dessas ações governamentais que envolvem fontes de financiamento, grupos profissionais e opinião pública, e que necessitam da constatação da sua efetiva contribuição na resolução de problemas sociais: uma estratégia que, a partir de modelos existentes em outros países, tem uma configuração teórica substantiva, mas que, na prática, distancia-se muito do modelo original. A avaliação do programa pode servir de guia para seu acompanhamento e fortalecimento, aproximando o modelo teórico da prática na atenção à saúde da população.

Observou-se no presente estudo um "efeito cascata" nas mazelas da Saúde da Família em João Pessoa. Os profissionais não têm vínculo empregatício, gerando rotatividade dos recursos humanos. Por conseguinte, há o reflexo da não capacitação dos profissionais que atuam na ESF, provocando incompatibilidade entre as diretrizes teóricas do SUS e o praticado na realidade da atenção à saúde por seus profissionais, que, muitas 
vezes, não têm o perfil para trabalho no SUS, comprometendo, portanto, a humanização. Junto à dificuldade de contratação de profissionais, surge a alta demanda de atendimentos clínicos para uma equipe que"não dá conta do serviço", afetando, portanto, os trabalhos de promoção de saúde e prevenção de doenças.

Reconhece-se, no entanto, que embora ainda tenha muito a melhorar no tocante às condições de trabalho dentro das ESF, João Pessoa avançou bastante nos últimos anos quando se observam os resultados de pesquisas anteriores (Oliveira, 2006; Sousa \& Souza Filho, 2008). Percebe-se um progresso na infraestrutura física e na distribuição de materiais, créditos esses dados, em especial, às Unidades Unificadas, modelos que vêm sendo implantados desde 2008. Caminha-se, entretanto, a passos lentos, havendo ainda muitas USF sem infraestrutura mínima, em casas improvisadas, situação que deve ser corrigida para melhorar os serviços de saúde à população. Portanto, após descoberto um modelo viável das Unidades Unificadas, faz-se necessário, agora, replicá- lo.

Os aspectos mais preocupantes da realidade da ESF em João Pessoa, entretanto, referem-se à eficiência no atendimento, à capacitação dos profissionais e ao sistema de referência e contra-referência. Verificou-se a necessidade de buscar estratégias de manutenção dos profissionais nas EqSF, diminuindo sua rotatividade através de incentivos financeiros e de vínculos empregatícios estáveis, e podendo, dessa forma, investir em capacitações permanentes para profissionais que permanecerão nos cargos e que reverterão esse conhecimento em melhoria de serviços à população. Faz-se necessário, ainda, o respeito aos princípios da integralidade e da hierarquização, tornando-se imprescindível a real integração entre os três níveis de assistência do Sistema Único de Saúde - primário, secundário e terciário -, devendo-se diminuir as burocracias no referenciamento, incentivando, inclusive, o retorno do usuário pela contra-referência.

Nesse sentido, montar uma rede intranet é a maneira mais rápida, barata e eficaz de suplantar as dificuldades de marcação e do sistema de referência e contra-referência. Deixar para o usuário a obtenção dos laudos e diagnósticos termina por culpabilizar a vítima, uma vez que são pessoas carentes tanto de recursos financeiros para os deslocamentos quanto de informação sobre os procedimentos.

\section{Referências}

Albuquerque, F. J. B. (1999). Apontamentos para uma Psicologia sócio-rural no Brasil. In I Congresso Norte e Nordeste de Psicologia. Salvador. Anais do I Congresso NorteNordeste de Psicologia (Vol.1, pp.12-17). Salvador: UFBA.

Barreira, . C. R. N. (2001). Tendências eperspectivas na avaliação de políticas e programas sociais. São Paulo: Cortez.

Beck, A. T. (1982). Terapia cognitiva da depressão. Rio de Janeiro: Zahar.

Belloni, l., Magalhães, H., \& Sousa, L. C. (2003). Metodologia de avaliação em políticas públicas (3a ed.). São Paulo: Cortez. (Coleção Questões da Nossa Época, v. 75).

Campbell, D. T. (1969). Reforms and experiments. American Psychologist, 4, 409-429.

Cano, I. (2004). Introdução à avaliação de programas sociais. Rio de Janeiro: FGV.

Cordioli, A. V. (2008). Psicoterapias (3a ed.). São Paulo: Editora Artmed.

Faleiros, J. J. (2003). para reorientar o modelo assistencial. quantos e quais? Revista Brasileira de Educação Médica, 27(1), 55-64.

Melo, C. F. (2009). Avaliação da estratégia saúde da família a partir das crenças de seus profissionais. Dissertação de mestrado não-publicada, Universidade Federal da Paraíba, João Pessoa.

Oliveira, S. F. (2006). Avaliação do programa saúde da família: uma análise a partir das crenças dos profissionais da equipe de saúde e da população assistida. Dissertação de mestrado não-publicada, Universidade Federal da Paraíba, João Pessoa.

Sousa, L. M., \& Souza Filho, E. A. (2008). Percepções sociais de pacientes sobre profissionais de saúde e outros estressores no ambiente de unidade de terapia intensiva. Estudos de Psicologia (Campinas), 25 (3), 333-342. doi: 10.15 90/S0103-166X2008000300002.

Recebido em: 30/4/2010

Versão final reapresentada em: 10/1/2011

Aprovado em: 12/1/2011 